\title{
Morpho-dynamic changes of mitochondria during ageing of human endothelial cells
}

\author{
Marina Jendrach ${ }^{\mathrm{a}, 1, *}$, Sandra Pohl ${ }^{\mathrm{a}, 1}$, Monika Vöth ${ }^{\mathrm{a}}$, Axel Kowald ${ }^{\mathrm{b}}$, \\ Peter Hammerstein ${ }^{c}$, Jürgen Bereiter-Hahn ${ }^{\mathrm{a}}$ \\ ${ }^{a}$ Kinematic Cell Research Group, JW Goethe University, Marie-Curie Strasse 9, 60439 Frankfurt/Main, Germany \\ ${ }^{\mathrm{b}}$ Kinetic Modelling Group, MPI Molecular Genetics, Berlin, Germany \\ ${ }^{\mathrm{c}}$ Institute for Theoretical Biology, Humboldt University Berlin, Germany
}

Received 23 December 2004; received in revised form 28 February 2005; accepted 4 March 2005

Available online 8 April 2005

\begin{abstract}
Mitochondrial morphology is regulated in many cultured eukaryotic cells by fusion and fission of mitochondria. A tightly controlled balance between fission and fusion events is required to ensure normal mitochondrial and cellular functions. During ageing, mitochondria are undergoing significant changes on the functional and morphological level. The effect of ageing on fusion and fission of mitochondria and consequences of altered fission and fusion activity are still unknown although theoretical models on ageing consider the significance of these processes. Human umbilical vein endothelial cells (HUVECs) have been established as a cell culture model to follow mitochondrial activity and dysfunction during the ageing process. Mitochondria of old and postmitotic HUVECs showed distinct alterations in overall morphology and fine structure, and furthermore, loss of mitochondrial membrane potential. In parallel, a decrease of intact mitochondrial DNA (mtDNA) was observed. Fission and fusion activity of mitochondria were quantified in living cells. Mitochondria of old HUVECs showed a significant and equal decrease of both fusion and fission activity indicating that these processes are sensitive to ageing and could contribute to the accumulation of damaged mitochondria during ageing.
\end{abstract}

(C) 2005 Elsevier Ireland Ltd. All rights reserved.

Keywords: Ageing; Mitochondria; Fusion; Fission

\section{Introduction}

Mitochondria are dynamic organelles, varying from long tubular networks to single spherical organelles. These frequent changes in number and morphology are due to mitochondrial fission and fusion (Bereiter-Hahn and Vöth, 1994). Fragmentation of mitochondria is a common event before cytokinesis and ensures equal distribution of mitochondria on the daughter cells (Barni et al., 1996). In many cultured vertebrate cells, fission and fusion of mitochondria take place all the time, and recently, it was shown that fission and fusion of mitochondria are also a

\footnotetext{
* Corresponding author. Tel.: +49 69 79829605; fax: +49 6979829607. E-mail address: jendrach@zoology.uni-frankfurt.de (M. Jendrach).

${ }^{1}$ Authors contributed equally to this work.
}

regular event in plant cells (Karbowski et al., 2004; Arimura et al., 2004). The molecular mechanisms underlying these processes in mammalian cells were intensively studied during the last years, and by now five essential proteins have been identified and possible models proposed (reviewed by Bossy-Wetzel et al., 2003). The physiological significance of the continual fusion and division of mitochondria is still under debate; a possible function for fusion could be a rescue mechanism for damaged mitochondria by exchange of mitochondrial DNA (mtDNA) and/or mitochondrial proteins.

The main task of mitochondria-production of ATP by the complexes of the respiratory chain-is also the reason why mitochondria are heavily featuring in apoptosis and ageing. Mitochondria are the primary source of reactive oxygen species (ROS), mainly from complexes I and III, and 
while ROS production is occurring throughout life it is increased in aged cells (Sastre et al., 2003; Turrens, 2003). According to the oxidative damage theory of ageing, first proposed by Harman (1972), mitochondria are also the main targets of ROS, since increased levels of ROS damage predominantly mitochondrial proteins, lipids and mitochondrial DNA. mtDNA is more sensitive than genomic DNA to ROS-induced damage, since it is not protected by histons and its repair capabilities are limited (reviewed by Wei and Lee, 2002).

The mitochondrial theory of ageing proposes that the age-related accumulation of defective mitochondria is the main mechanism underlying the ageing process (Linnane et al., 1989). A critical aspect is to understand how damaged mitochondria can out compete the wild type. Three possible explanations have been suggested: (i) accumulation by random drift of selection neutral mutations (Elson et al., 2001), (ii) faster replication of deletion mutants because of their reduced size (Wallace, 1992) and (iii) slower degradation of defective organelles (SOS hypothesis) (de Grey, 1997; Kowald and Kirkwood, 2000). While the first two theories require mitochondrial fusion, the SOS hypothesis is based on a lack of fusion activity.

Our current study therefore aims to provide experimental data that evaluate the role of mitochondrial fusion and fission in ageing. With human umbilical vein endothelial cells (HUVECs) as a model, we investigated the changes in young and proliferative compared to old and postmitotic cells with special regard to the fission and fusion activity. We have quantified fission and fusion events on the functional level and for the first time we can show that this activity is significantly reduced in postmitotic cells together with other age-dependent parameters, like mitochondrial membrane potential and intact mtDNA.

\section{Experimental procedures}

\subsection{Isolation and culture of HUVECs}

Primary human endothelial cells were isolated from the umbilical cord under sterile conditions. The vein from the umbilical cord was washed with PBS. Afterwards, the vein was clamped and incubated for $10 \mathrm{~min}$ in basal medium with dispase $(100 \mathrm{U} / \mathrm{ml})$. The resulting endothelial cell solution was rinsed with basal medium in a tube and centrifuged. The cells were resuspended in growth medium and cultivated at $37{ }^{\circ} \mathrm{C}$ and $5 \% \mathrm{CO}_{2}$. Basal medium consisted of M199 with heparin (10 IE), hepes buffer $(20 \mathrm{mM})$ and gentamycin $(100 \mu \mathrm{g})$. Growth medium contained basal medium with $10 \%$ FCS, $10 \%$ human serum and ECGF $(20 \mu \mathrm{g} / \mathrm{ml})$.

In parallel, HUVECs were pursued form Promocell (C12200) and cultivated in Endothelial Cell Growth Medium (Promocell \#C-22010).
Doubling time of cells was determined by regular counting. Factor VIII expression was monitored by fluorescence microscopy: first antibody was mouse anti human factor VIII (DAKO, dilution 1:100) and second antibody was goat anti-mouse Cy3 (Dianova, dilution 1:100). Cultures with a doubling time of more than $100 \mathrm{~h}$ and factor VIII expression $<70 \%$ of that of first passages were classified as old and postmitotic cells.

\subsection{Quantification of fission and fusion events}

HUVECs were cultivated for 2 days on a coverslip. Mitochondria in the peripheral region of a cell were filmed for $10 \mathrm{~min}$ in phase contrast with a microscope (Zeiss) and a CCD-camera (PCO), one frame per second. Single images were assembled to a video sequence (Quick Time Player). The fission and fusion events were counted and related to the area of mitochondria (in $\mu \mathrm{m}^{2}$ ). The mitochondria from the first image of the video sequence were copied with GIMP and determination of the area was done with Image $\mathbf{J}$.

\subsection{Confocal microscopy}

Mitochondria were visualised by staining with Mitotracker Green or Mitotracker Red ${ }^{\mathbb{R}}$ (Molecular Probes) in a final concentration of $250 \mathrm{nM}$ for $1 \mathrm{~h}$. Microtubules were stained with antibody YL as primary antibody (personal gift of J. Wehland) and anti-rat Cy3 as secondary antibody (Dianova). For dynactin staining primary antibody was against $\mathrm{p} 150^{\text {Glued }}$ (BD Biosciences) and second antibody was anti-mouse FITC (Dianova). For staining of mtDNA, cells were treated with Picogreen (Molecular Probes), final dilution 1:100. For determination of mitochondrial membrane potential, cells were treated with dimethylaminostyrylmethyl-pyridinium-iodine (DASPMI) (personal gift of Bayer AG to J.B.H.) for $1 \mathrm{~h}$. Micrographs were taken with a Leica TCS 4D confocal laser scanning microscope fitted with the appropriate filters and PL Fluotar objective $(100 \times$, $1.3 \mathrm{NA}$ ) that was controlled by the SCAN Ware 5.10 software (Leica, Wetzlar, Germany). Live cell experiments were performed at $37^{\circ} \mathrm{C}$ and $5 \% \quad \mathrm{CO}_{2}$ in a humidified chamber.

\subsection{Electron microscopy}

Cells were fixed with phosphate-buffered $2.5 \%$ glutaraldehyde with $0.1 \mathrm{M}$ sodium phosphate for $1 \mathrm{~h}$. Then they were treated with $1 \%$ osmium tetroxid in hydrogen peroxide for $1 \mathrm{~h}$, dehydrated with acetone and embedded with epon. Electron microphotographs were taken with a Hitachi 500 transmission electron microscope (Hitachi).

\subsection{Quantification of $m t D N A$}

Total DNA was isolated from a total of $1 \times 10^{6}$ cells with the Flexi Gene DNA Kit (Qiagen). A 50 ng of total DNA was 

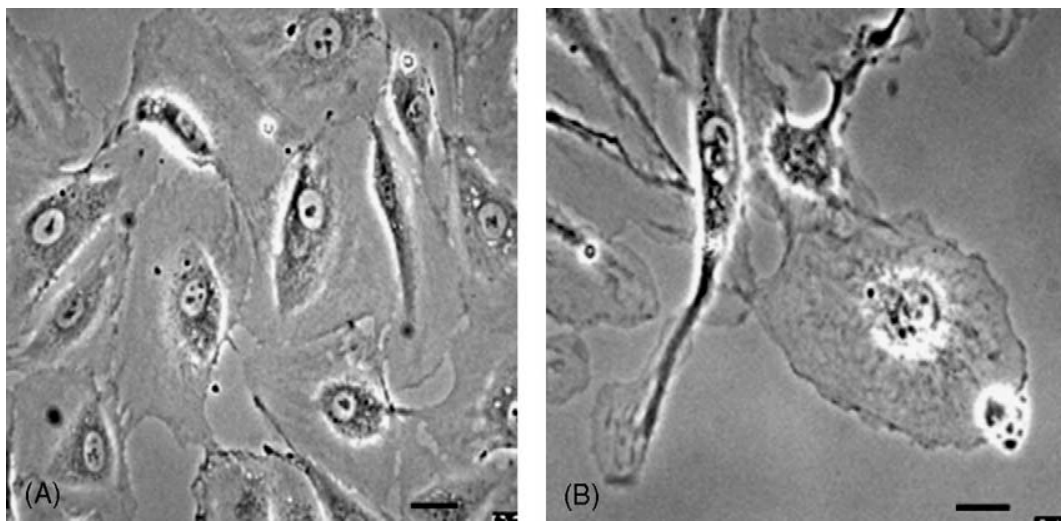

Fig. 1. Proliferative and postmitotic HUVECs in phase contrast. Young cells were proliferating cells with a generation time of 24-100 h and $100-70 \%$ of the cells expressed von Willebrand factor (A). Postmitotic cells were characterised by a generation time exceeding $100 \mathrm{~h}$ and an expression of von Willebrand factor in less than $70 \%$ of the cells (B). Scale bar $=10 \mu \mathrm{m}$.

used for PCR reactions. The $220 \mathrm{bp}$ and $8.9 \mathrm{kbp}$ fragments of mtDNA were amplified by PCR according to Santos et al. (2002). After gel electrophoresis quantification was archived with Easywin 32 software (Herolab).

\subsection{Statistics}

Results are expressed as means \pm S.E.M. of $n$ observations. Student's $t$-test was used for significance analysis. Differences were considered statistically significant when $P<0.01$

\section{Results}

\subsection{Ageing of HUVECs}

HUVECs were used as a model system to study age-related effects. During cultivation the doubling time of each passage and the expression of factor VIII (von Willebrand factor) was analysed. Presence of factor VIII has been shown to decrease during ageing of endothelial cells and can be used as marker for ageing (Kiyonaga et al., 2001). Consequently, HUVECs with a doubling time of more than $100 \mathrm{~h}$ and less than $70 \%$ factor VIII expression compared to young and proliferative cells were classified as old and postmitotic.

In phase contrast microscopy, young HUVECs showed the typical polygonal "cobblestone" phenotype of endothelial cells (Fig. 1A). However, postmitotic cells showed an altered morphology. These cells were much larger than mitotic cells and some cells were multinuclear. Many postmitotic HUVECs showed long filopodia-like extensions and cells appeared in general more heterogeneous than proliferating cells. The thickness of postmitotic cells in the $z$-axis differed; some regions were flat and in some parts cytoplasm and mitochondria accumulated (Fig. 1B).

\subsection{Changes in mitochondria of old and postmitotic HUVECs}

To analyse the mitochondria of young proliferative and old postmitotic HUVECs, confocal microscopy was used after staining of mitochondria with Mitotracker Red ${ }^{\circledR}$. In young HUVECs, mitochondria were distributed evenly throughout the cytoplasm and consisted mostly of long, single tubules (Figs. 2A and 6A). In contrast, most

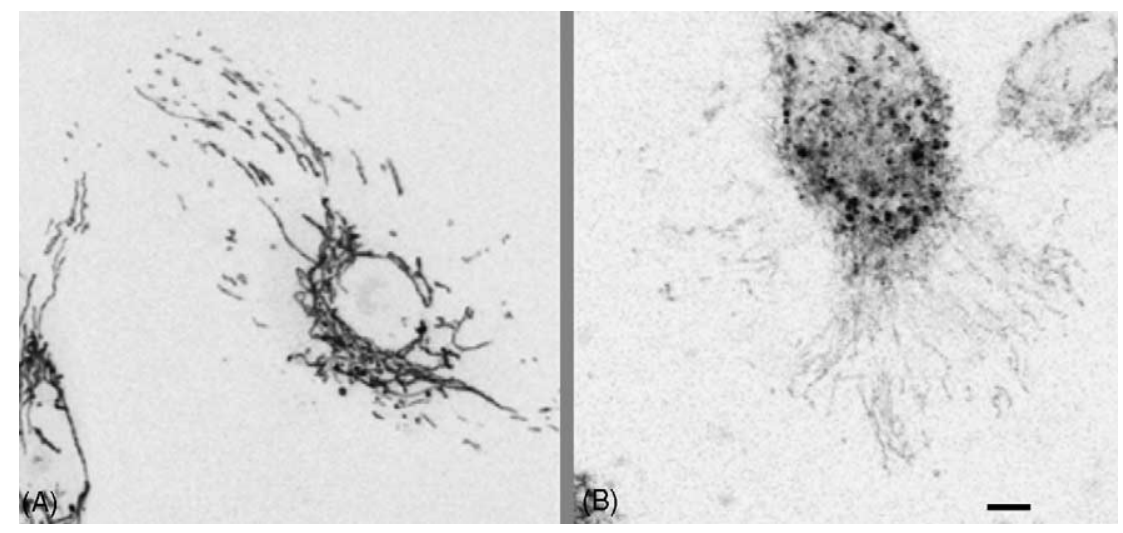

Fig. 2. HUVECs were stained with mitochondrial membrane potential-sensitive dye DASPMI. Young HUVEC had a strong DASPMI fluorescence (A). Mitochondria in postmitotic cells (B) were accumulated in the perinuclear area and showed mostly a diminished membrane potential. Scale bar $=10 \mu \mathrm{m}$. 

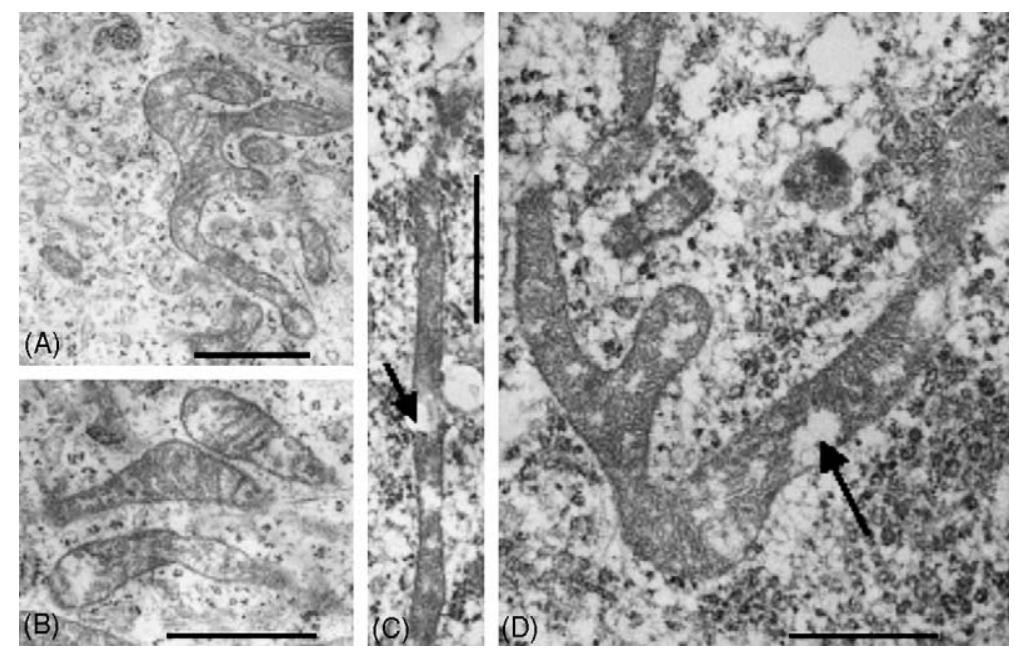

Fig. 3. Electron microscope images of young and old HUVECs show distinct changes in the fine structure of mitochondria. Cristae in mitochondria of proliferative cells (A and B) were numerous and clearly structured. In contrast, in old cells (C and D) many cristae were degenerated, barely visible and mitochondria showed swollen regions devoid of cristae (arrow). Scale bar $=1 \mu \mathrm{m}$.

mitochondria of postmitotic cells were localised around the nucleus and appeared aggregated. Nevertheless, in the cell periphery still some tubular, "normal looking" mitochondria could be found (Figs. 2B and 6C).

To examine if these morphological changes are reflected by physiological alterations, HUVECs were stained with dimethylaminostyrylmethyl-pyridinium-iodine (DASPMI) and analysed by confocal microscopy. Uptake of DASPMI depends on the mitochondrial membrane potential (BereiterHahn, 1976); therefore, we used this dye to compare the membrane potential of proliferative and postmitotic cells. Mitochondria of young HUVECs displayed a strong and almost evenly distributed DASPMI fluorescence (Fig. 2A). In postmitotic cells, however, mitochondria of most cells were only weakly stained while the majority of the dye remained in the cytoplasm, indicating a loss of mitochondrial membrane potential during ageing (Fig. 2B).

In the electron microscope, mitochondria of young and mitotically active HUVECs show clearly defined and wellordered cristae (Fig. 3A and B). Mitochondria of postmitotic cell cultures contained fewer cristae, which seemed to be less clearly structured and organised; in some regions cristae were difficult to recognise. In the matrix of postmitotic mitochondria, distinct swollen areas were found (arrows) that contained no cristae at all (Fig. 3C and D). Thus, the loss of membrane potential in ageing cells was accompanied by alterations in the fine structure of the organelles.

\subsection{Fission and fusion of mitochondria were reduced in postmitotic cells}

Since theoretical models of mitochondrial ageing depend on the presence or absence of mitochondrial fission and fusion activity, we established a method to quantify mitochondrial fission and fusion in living cells. Mitochondria in the periphery of proliferative and postmitotic cell cultures were filmed and the number of fission and fusion events in $10 \mathrm{~min} / 100 \mu \mathrm{m}^{2}$ mitochondria area was evaluated (Fig. 4). Mitochondria that stayed joined while they are moving were defined as fused. We have already shown by correlative electron microscopy that this kind of mitochondria was fused with the outer and inner mitochondrial membrane (Bereiter-Hahn and Vöth, 1994). Furthermore, we observed in this kind of fused mitochondria exchange of proteins of the inner mitochondrial membrane (Jendrach et al., unpublished results). Filming was restricted to the cell periphery because of the perinuclear accumulation of mitochondria in postmitotic cells.

In young proliferative cells, fusion and fission of mitochondria were almost equal with $2.19( \pm 0.19)$ fusion

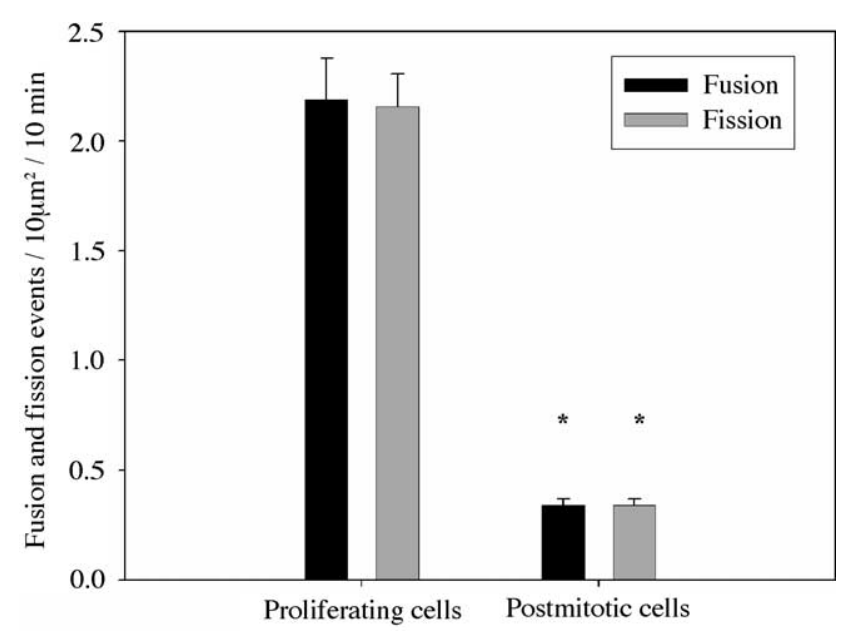

Fig. 4. Quantification of mitochondrial fission and fusion events in young and old HUVECs. Mitochondria in the peripheral region of proliferating and postmitotic cells were filmed and fusion and fission events were determined as described in experimental procedures. Fission and fusion events in $10 \mathrm{~min} / 100 \mu \mathrm{m}^{2}$ mitochondria area were evaluated in proliferative $(n=34)$ and postmitotic $(n=23)$ HUVECs. The amount of both fusion and fission events was significantly decreased in old cells $(P<0.01)$. 
events in $10 \mathrm{~min} / 100 \mu \mathrm{m}^{2}$ mitochondria area and 2.16 $( \pm 0.15)$ fission events in $10 \mathrm{~min} / 100 \mu \mathrm{m}^{2}$ mitochondria area. In contrast, fission and fusion rates were significantly reduced in old cells although still equal with an average of $0.34( \pm 0.02)$ fusion events in $10 \mathrm{~min} / 100 \mu \mathrm{m}^{2}$ mitochondria area and $0.34( \pm 0.02)$ fission events in $10 \mathrm{~min} / 100 \mu \mathrm{m}^{2}$ mitochondria area. Furthermore, mitochondria in young cells were quite motile, while in postmitotic cells they moved less and at slower speed.

\subsection{Reduction of mitochondrial dynamics in postmitotic HUVECs is not related to changes in the cytoskeleton}

Diminished mobility and reduced fission and fusion activity of mitochondria in old cells could result from an altered amount of cytoskeletal elements responsible for transporting mitochondria. Mitochondria are mainly moving along microtubules (Tanaka et al., 1998). Therefore, microtubules of proliferative and postmitotic cells were visualised by antibody staining. Fluorescence micrographs of young cells exhibited well-ordered and regular microtubules (Fig. 5B). In old cells, no reduction of the microtubule content or distribution has been observed, the microtubule network was still well spread, reaching up to the periphery of the cells. In some regions, in particular, close to the nucleus, increased microtubule density and bundling was found (Fig. 5D). This is most probably due to the fact that in these regions of the cell cytoplasm and organelles were concentrated (see Fig. 1B).

Since microfilaments are probably involved in situating mitochondria, F-actin distribution has been investigated by phalloidin staining. Postmitotic cells contained noticeably more stress fibers and by no means F-actin content was reduced (data not shown).

Disruption of the dynein/dynactin complex has been reported to cause perinuclear clustering of mitochondria (Varadi et al., 2004), thus expression of the dynactin subunit p $150^{\text {Glued }}$ was investigated. Both microtubules of proliferating and postmitotic HUVECs co-localised with a dense dynactin staining, demonstrating that expression levels of dynactin were not changed during ageing (Fig. 5A and C). Also, blocking of kinesin-mediated transport resulted in perinuclear clustering of mitochondria (de Vos et al., 2000) but analysis of kinesin (KIF3A) expression in young and old HUVECs by confocal microscopy also showed no downregulation during ageing (data not shown).

\subsection{Mitochondria of old HUVECs showed loss of intact $m t D N A$}

mtDNA can be visualised in living cells using Picogreen, a fluorochome which selectively binds to nucleic acids with
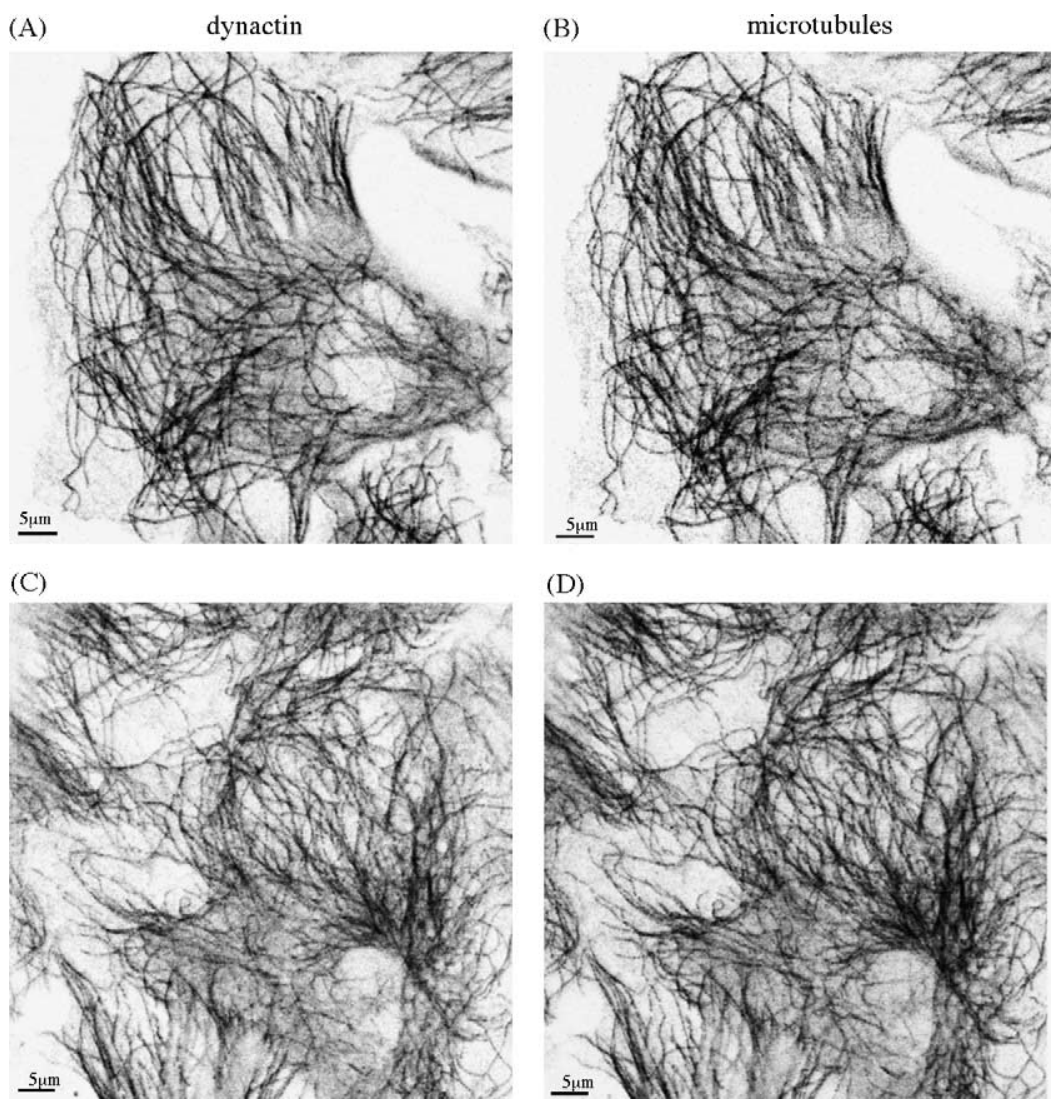

Fig. 5. Microtubule and dynactin expression were not changed during ageing. Young (A and B) and old (C and D) HUVECs were analysed. Dynactin was stained with an antibody against the $150^{\text {Glued }}$ subunit $(A$ and C) and microtubules were visualised with antibody YL $(B$ and D). Scale bar $=5 \mu m$. 

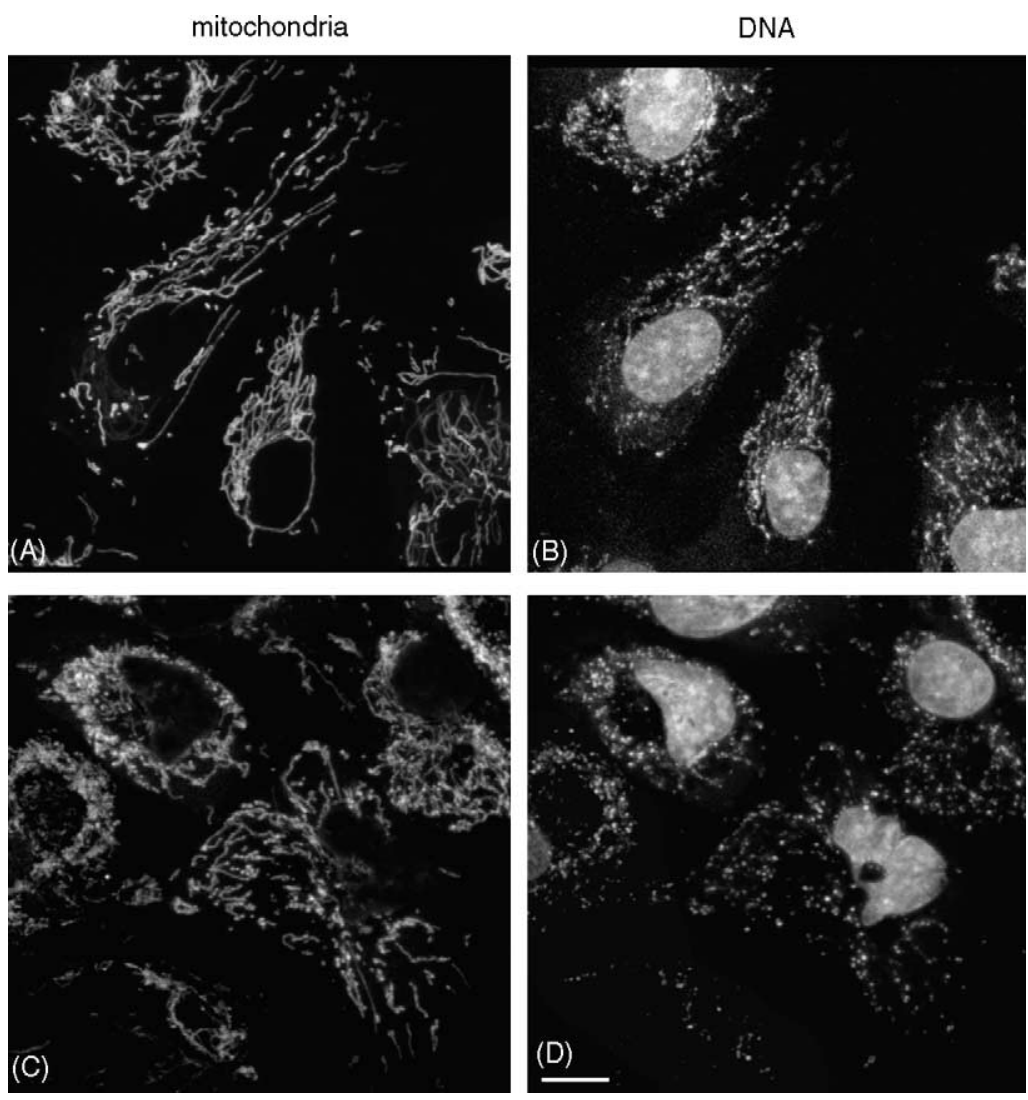

Fig. 6. Visualisation of mitochondrial DNA (mtDNA) in young (A and B) and old (C and D) HUVECs. Mitochondria in (A and C) were stained with Mitotracker Red ${ }^{\circledR}$. Additionally both nuclei and mtDNA were stained with Picogreen (B and D). Comparison of Picogreen staining in (B and D) shows much less mitochondrial nucleoids with large spaces in between in senescent cells (D). Scale bar $=10 \mu \mathrm{m}$.

higher affinity for DNA than for RNA (Bereiter-Hahn and Vöth, 1996). Nuclei as well as mitochondrial nucleoids in proliferating and postmitotic HUVECs were detected by this method (Fig. 6B and D). Most mitochondria of young cells

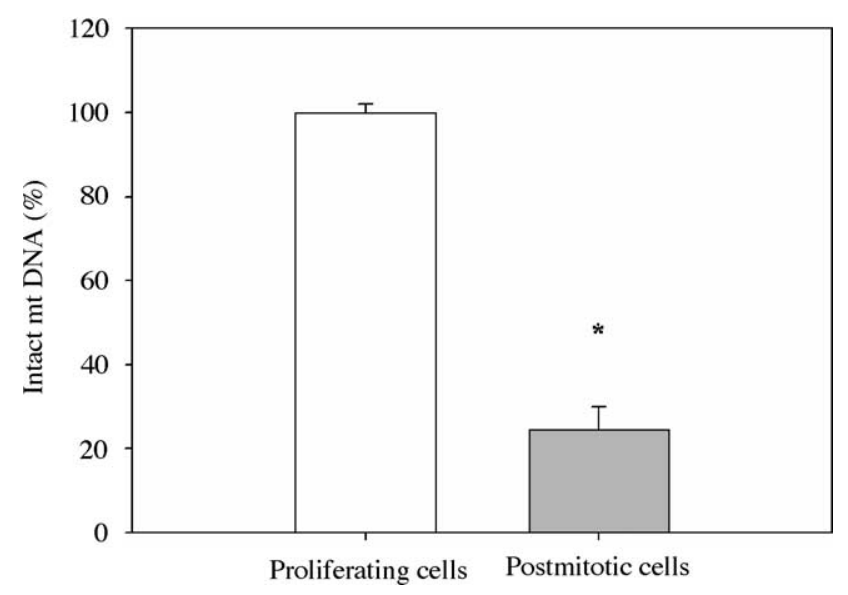

Fig. 7. Quantification of intact mtDNA in proliferative and postmitotic HUVECs: total DNA from young and old HUVECs was isolated and a small $(220 \mathrm{bp})$ and a large $(8.9 \mathrm{kbp})$ fragment of mtDNA were amplified by semiquantitative PCR as described in experimental procedures. The small fragment was used for normalisation of total mtDNA content and content of intact mtDNA of young HUVECs was set to $100 \%$. The amount of intact mtDNA in postmitotic cells was significantly decreased compared to young cells $(n=8 ; P<0.01)$. contained several strongly stained mitochondrial nucleoids (Fig. 6B), while in mitochondria of postmitotic cells less and faintly fluorescing nucleoids appeared (Fig. 6D). Since short fragments of mtDNA exhibit low fluorescence only, the Picogreen staining of postmitotic cells can be interpreted as loss as well as extensive fragmentation of mtDNA in the course of ageing.

To quantify the apparent reduction of intact mtDNA, total DNA from young and old HUVECs was isolated and a small (220 bp) and a large $(8.9 \mathrm{kbp})$ fragment of mtDNA were amplified in a semiquantitative PCR, according to Santos et al. (2002), whereas the small fragment was used for normalisation. In old HUVECs, amplification of the large mtDNA fragment was reduced by $75.4 \%( \pm 15.2 \%)$ when compared to mitotically active young cultures, emphasising a strong loss of intact mtDNA during ageing (Fig. 7).

\section{Discussion}

Primary human endothelial cells have been established as a model for cell ageing (Kiyonaga et al., 2001; Hampel et al., 2004). HUVECs pass from a young and proliferative state to an old and postmitotic state during a relatively short time and represent therefore a good model to study organelle-related ageing processes. Postmitotic HUVECs are characterised by 
an extremely extended generation time and reduced factor VIII (von Willebrand factor) expression (Kiyonaga et al., 2001; our own data). Mitochondria of postmitotic cells showed distinct alterations in morphology and, at the functional level, a reduction of fusion and fission activity, a decline of mitochondrial membrane potential and an increased amount of deletions in the mtDNA.

Fission and fusion of mitochondria are frequent in several vertebrate cells in culture (Bereiter-Hahn and Vöth, 1994; Karbowski et al., 2004). For the first time, a real-time quantification of mitochondrial fusion and fission in living cells, comparing young and postmitotic cells, was performed. The number of fission events equalled that of fusion events in young and old cells, although the total number of events was strongly decreased in postmitotic cultures. This behaviour indicates a tight control of fission and fusion processes. Until now three proteins involved in mitochondrial fusion (Mfn1, Mfn2 and Opa1) and two participating in fission (Fis1 and Drp1) of mammalian cells have been described, all nucleus-encoded (reviewed by Bossy-Wetzel et al., 2003). Overexpression or downregulation of one of these proteins results in dramatic changes in mitochondrial morphology and organisation (Eura et al., 2003; Olichon et al., 2003; Frieden et al., 2004; Stojanovski et al., 2004). These results are stressing the need for a balanced rate of fission and fusion events.

In postmitotic HUVECs, a strong reduction of fission and fusion events has been observed. We did only analyse mitochondria in the periphery of the cells, due to perinuclear mitochondrial clustering. All three versions of the mitochondrial theory of ageing - random drift, faster replication and slower degradation (SOS theory) - have to consider mitochondrial fusions. The SOS theory states that defective mitochondria might be degraded slower than healthy mitochondria, which could result in their accumulation (de Grey, 1997; Kowald and Kirkwood, 2000). A tight link between genotype and phenotype is important for this proposal. Mitochondrial fusion events destroy such a link, since they allow for a mixing of mitochondrial proteins and DNA between fused mitochondria. Our results of a high fusion rate in young cells and a low fusion rate in old cells (Fig. 4), point to a more complex picture. In young cells, defective mitochondria may not accumulate via a SOS mechanism, while with age (and concomitantly reduced fusion rate) the SOS idea might become more significant.

In old and postmitotic cells, most mitochondria were clustered around the nucleus and appeared to be less mobile than in young cells. The aggregation of mitochondria in the perinuclear region of postmitotic cells can have different reasons. Perinuclear clustering of mitochondria has been observed in apoptosis as well as in necrosis (Thomas et al., 2000; Li et al., 2004) but we did not observe any caspase-3 activation in adherent postmitotic cells (data not shown), indicating that cells were not undergoing apoptosis. Overexpression of fusion proteins Mfn1 and Mfn2 in Hela cells also resulted in clustering of interconnected mitochondria
(Eura et al., 2003). An increased or reduced expression of one of the fission- or fusion-initiating proteins, however, is ruled out in our opinion since fission and fusion activity remained balanced in old cells. Mitochondria in mammalian cells are moved mainly along microtubules (Tanaka et al., 1998; de Vos et al., 2000). The dynein/dynactin complex is involved in recruitment of fission protein Drp1 and disruption of this complex resulted in perinuclear clustering of mitochondria (Varadi et al., 2004). The same effect was achieved by inhibition of kinesin (de Vos et al., 2000). In postmitotic HUVEC, the microtubule and F-actin content and organisation did not differ from young cells and also the amount of dynactin and kinesin bound to microtubules was comparable in young and old cells, indicating that neither the cytoskeleton nor the motor protein expression was reduced during ageing, although the activity of motor proteins could be varying between proliferating and postmitotic cells.

In many mitochondria of old and postmitotic HUVECs, a diminished mitochondrial membrane potential was observed. This is consistent with reports of lowered mitochondrial membrane potential in different aged cell types and tissues (Hagen et al., 1997; Mather and Rottenberg, 2002; Xiong et al., 2004). Hutter et al. (2004) observed a reduced membrane potential in a subpopulation of aged fibroblasts and an accumulation of mitochondria with low membrane potential in postmitotic cells. Furthermore, in electron micrographs of postmitotic HUVEC mitochondria showed degeneration and loss of the cristae structures, indicating a reduced amount of available inner mitochondrial membrane. A diminished expression of respiratory chain complexes could be a consequence of these structural changes, resulting in a reduced membrane potential. Similar changes in the fine structure of mitochondria have been observed in smooth muscle cells from aged rats, where also a lowered mitochondrial membrane potential was measured (Lopes et al., 2004). Taken together the reduced number of fission and fusion events, the diminished mobility and clustering of mitochondria could be an effect of decreased energy production in ageing cells.

Furthermore, a diminished ATP content in postmitotic cells could be attributed at least partially to the increased number of mutations in the mtDNA during ageing (reviewed by Wei and Lee, 2002). In our system, we observed, by fluorescence microscopy, a diminished mtDNA signal in mitochondria of postmitotic cells. Semiquantitative PCR revealed a significant loss of intact mtDNA. Although we did not check for the typical deletions common in ageing cells, the PCR amplification product of the mtDNA genome (5999-14882) encompasses the region where many deletions occur (Trounce et al., 1989; Katayama et al., 1991; Zhang et al., 1992). To our knowledge, this is the first study showing this kind of changes in the mtDNA of HUVEC cells during ageing.

Fusion of mitochondria could possibly act as a rescue mechanism for damaged mitochondria (Bossy-Wetzel et al., 
2003). In cell culture systems, mitochondrial fusion can complement mtDNA mutations by exchange of mtDNA or transcripts (Ono et al., 2001). Also, proteins of the inner membrane and matrix components can be exchanged during fusion of mitochondria (Bereiter-Hahn and Vöth, 1994; Jendrach et al., unpublished results). The occurrence and impact of fusion and fission in vivo is still under discussion. Seo et al. (2004) found an uneven distribution of a recombinant protein of the inner mitochondrial membrane in muscle cells and neurons of transgenic mice, indicating a lack of exchange between mitochondria in vivo. On the other hand, compensation of mtDNA defects was observed in vivo in kidney, heart and skeletal muscle cells of transgenic mice, this, as concluded by the authors, being a consequence of intermitochondrial complementation (Nakada et al., 2001; Sato et al., 2004). Currently, the consequences of reduced numbers of fusion and fission events during ageing of HUVECs are difficult to predict. If fusion is really utilised to rescue damaged mitochondria, the reduced amount of fission and fusion events in old cells could result in a diminished exchange of mtDNA and/or proteins and hereby possible contributing to the accumulation of damaged mitochondria, while in young cells mitochondrial defects may be compensated by higher fusion rates. Further experiments in the earlier stages of ageing should determine the contribution of fission and fusion to the ageing process.

\section{Acknowledgements}

This work was supported by a grant from DFG BE423/ 23-2. Furthermore important support was received from EU Integrated Project MiMage CT 2004-512020.

\section{References}

Arimura, S., Yamamoto, J., Aida, G.P., Nakazono, M., Tsutsumi, N., 2004. Frequent fusion and fission of plant mitochondria with unequal nucleoid distribution. Proc. Natl. Acad. Sci. 101, 7805-7808.

Barni, S., Sciola, L., Spano, A., Pippia, P., 1996. Static cytofluorometry and fluorescence morphology of mitochondria and DNA in proliferating fibroblasts. Biotech. Histochem. 71, 66-70.

Bereiter-Hahn, J., 1976. Dimethylaminostyrylmethyl-pyridiniumiodine (DASPMI) as a fluorescent probe for mitochondria in situ. Biochim. Biophys. Acta. 423, 1-14.

Bereiter-Hahn, J., Vöth, M., 1994. Dynamics of mitochondria in living cells: shape changes, dislocations, fusion, and fission of mitochondria. Microsc. Res. Tech. 27, 198-219.

Bereiter-Hahn, J., Vöth, M., 1996. Distribution and dynamics of mitochondrial nucleoids in animal cells in culture. Exp. Biol. online 1, 4.

Bossy-Wetzel, E., Barsoum, M.J., Godzik, A., Schwarzenbacher, R., Lipton, S.A., 2003. Mitochondrial fission in apoptosis, neurodegeneration and aging. Curr. Opin. Cell. Biol. 15, 706-716.

de Grey, A.D., 1997. A proposed refinement of the mitochondrial free radical theory of aging. Bioessays 19, 161-166.

de Vos, K., Severin, F., Van Herreweghe, F., Vancompernolle, K., Goossens, V., Hyman, A., Grooten, J., 2000. Tumor necrosis factor induces hyperphosphorylation of kinesin light chain and inhibits kinesinmediated transport of mitochondria. J. Cell. Biol. 149, 1207-1214.

Elson, J.L., Samuels, D.C., Turnbull, D.M., Chinnery, P.F., 2001. Random intracellular drift explains the clonal expansion of mitochondrial DNA mutations with age. Am. J. Hum. Genet. 68, 802-806.

Eura, Y., Ishihara, N., Yokota, S., Mihara, K., 2003. Two mitofusion proteins, mammalian homologues of $\mathrm{FZO}$, with distinct functions are both required for mitochondrial fusion. J. Biochem. 134, 333-344.

Frieden, M., James, D., Castelbou, C., Danckaert, A., Martinou, J.C., Demaurex, N., 2004. $\mathrm{Ca}(2+)$ homeostasis during mitochondrial fragmentation and perinuclear clustering induced by hFis1. J. Biol. Chem. 279, 22704-22714.

Hagen, T.M., Yowe, D.L., Bartholomew, J.C., Wehr, C.M., Do, K.L., Park, J.Y., Ames, B.N., 1997. Mitochondrial decay in hepatocytes from old rats: membrane potential declines, heterogeneity and oxidants increase. Proc. Natl. Acad. Sci. 94, 3064-3069.

Hampel, B., Malisan, F., Niederegger, H., Testi, R., Jansen-Dürr, P., 2004. Differential regulation of apoptotic cell death in senescent human cells. Exp. Gerontol. 39, 1713-1721.

Harman, D., 1972. The biological clock: the mitochondria? J. Am. Geriatr. Soc. 20, 145-147.

Hutter, E., Renner, K., Pfister, G., Stockl, P., Jansen-Durr, P., Gnaiger, E., 2004. Senescence-associated changes in respiration and oxidative phosphorylation in primary human fibroblasts. Biochem. J. 380, 919-928.

Karbowski, M., Arnoult, D., Chen, H., Chan, D.C., Smith, C.L., Youle, R.J., 2004. Quantitation of mitochondrial dynamics by photolabeling of individual organelles shows that mitochondrial fusion is blocked during the Bax activation phase of apoptosis. J. Cell. Biol. 164, 493-499.

Katayama, M., Tanaka, M., Yamamoto, H., Ohbayashi, T., Nimura, Y., Ozawa, T., 1991. Deleted mitochondrial DNA in the skeletal muscle of aged individuals. Biochem. Int. 25, 47-56.

Kiyonaga, H., Doi, Y., Karasaki, Y., Arashidani, K., Itoh, H., Fujimoto, S., 2001. Expressions of endothelin-1, fibronectin, and interleukin-1 alpha of human umbilical vein endothelial cells under prolonged culture. Med. Electron. Microsc. 34, 41-53.

Kowald, A., Kirkwood, T.B., 2000. Accumulation of defective mitochondria through delayed degradation of damaged organelles and its possible role in the ageing of postmitotic and dividing cells. J. Theor. Biol. 202, $145-160$.

Li, J., Li, Q., Xie, C., Zhou, H., Wang, Y., Zhang, N., Shao, H., Chan, S.C., Peng, X., Lin, S.C., Han, J., 2004. Beta-actin is required for mitochondria clustering and ROS generation in TNF-induced, caspase-independent cell death. J. Cell Sci. 117, 4673-4680.

Linnane, A.W., Marzuki, S., Ozawa, T., Tanaka, M., 1989. Mitochondrial DNA mutations as an important contributor to ageing and degenerative disease. Lancet i, 642-645.

Lopes, G.S., Mora, O.A., Cerri, P., Faria, F.P., Jurkiewicz, N.H., Jurkiewicz, A., Smaili, S.S., 2004. Mitochondrial alterations and apoptosis in smooth muscle from aged rats. Biochim. Biophys. Acta 1658, 187-194.

Mather, M.W., Rottenberg, H., 2002. The inhibition of calcium signaling in $\mathrm{T}$ lymphocytes from old mice results from enhanced activation of the mitochondrial permeability transition pore. Mech. Ageing Dev. 123, 707-724.

Nakada, K., Inoue, K., Ono, T., Isobe, K., Ogura, A., Goto, Y.I., Nonaka, I., Hayashi, J.I., 2001. Inter-mitochondrial complementation: mitochondria-specific system preventing mice from expression of disease phenotypes by mutant mtDNA. Nat. Med. 7, 934-940.

Olichon, A., Baricault, L., Gas, N., Guillou, E., Valette, A., Belenguer, P., Lenaers, G., 2003. Loss of OPA1 perturbates the mitochondrial inner membrane structure and integrity, leading to cytochrome $c$ release and apoptosis. J. Biol. Chem. 278, 7743-7746.

Ono, T., Isobe, K., Nakada, K., Hayashi, J.I., 2001. Human cells are protected from mitochondrial dysfunction by complementation of DNA products in fused mitochondria. Nat. Genet. 28, 272-275.

Santos, J.H., Mandavilli, B.S., Van Houten, B., 2002. Measuring oxidative mtDNA damage and repair using quantitative PCR. Methods Mol. Biol. 197, 159-176. 
Sastre, J., Pallardo, F.V., Vina, J., 2003. The role of mitochondrial oxidative stress in aging. Free Radic. Biol. Med. 35, 1-8.

Sato, A., Nakada, K., Shitara, H., Yonekawa, H., Hayashi, J., 2004. In vivo interaction between mitochondria carrying mtDNAs from different mouse species. Genetics 167, 1855-1861.

Seo, B.B., Nakamaru-Ogiso, E., Cruz, P., Flotte, T.R., Yagi, T., MatsunoYagi, A., 2004. Functional expression of the single subunit NADH dehydrogenase in mitochondria in vivo: a potential therapy for complex I deficiencies. Hum. Gene Ther. 15, 887-895.

Stojanovski, D., Koutsopoulos, O.S., Okamoto, K., Ryan, M.T., 2004. Levels of human Fis1 at the mitochondrial outer membrane regulate mitochondrial morphology. J. Cell. Sci. 117, 1201-1210.

Tanaka, Y., Kanai, Y., Okada, Y., Nonaka, S., Takeda, S., Harada, A., Hirokawa, N., 1998. Targeted disruption of mouse conventional kinesin heavy chain, kif5B, results in abnormal perinuclear clustering of mitochondria. Cell 93, 1147-1158.

Thomas, W.D., Zhang, X.D., Franco, A.V., Nguyen, T., Hersey, P., 2000. TNF-related apoptosis-inducing ligand-induced apoptosis of melanoma is associated with changes in mitochondrial membrane potential and perinuclear clustering of mitochondria. J. Immunol. 165, 5612-5620.
Trounce, I., Byrne, E., Marzuki, S., 1989. Decline in skeletal muscle mitochondrial respiratory chain function: possible factor in ageing. Lancet 1 (8639), 637-639.

Turrens, J.F., 2003. Mitochondrial formation of reactive oxygen species. J. Physiol. 552, 335-344.

Varadi, A., Johnson-Cadwell, L.I., Cirulli, V., Yoon, Y., Allan, V.J., Rutter, G.A., 2004. Cytoplasmic dynein regulates the subcellular distribution of mitochondria by controlling the recruitment of the fission factor dynamin-related protein-1. J. Cell. Sci. 117, 4389-4400.

Wallace, D.C., 1992. Mitochondrial genetics: a paradigm for aging and degenerative diseases? Science 256, 628-632.

Wei, Y.H., Lee, H.C., 2002. Oxidative stress, mitochondrial DNA mutation, and impairment of antioxidant enzymes in aging. Exp. Biol. Med. 227, 671-682.

Xiong, J., Camello, P.J., Verkhratsky, A., Toescu, E.C., 2004. Mitochondrial polarisation status and $[\mathrm{Ca} 2+] i$ signalling in rat cerebellar granule neurones aged in vitro. Neurobiol. Aging 25, 349-359.

Zhang, C., Baumer, A., Maxwell, R.J., Linnane, A.W., Nagley, P., 1992. Multiple mitochondrial DNA deletions in an elderly human individual. FEBS Lett. 297, 34-38. 